\title{
Spektrum Pneumologie - wissenswert, kompakt, anregend
}

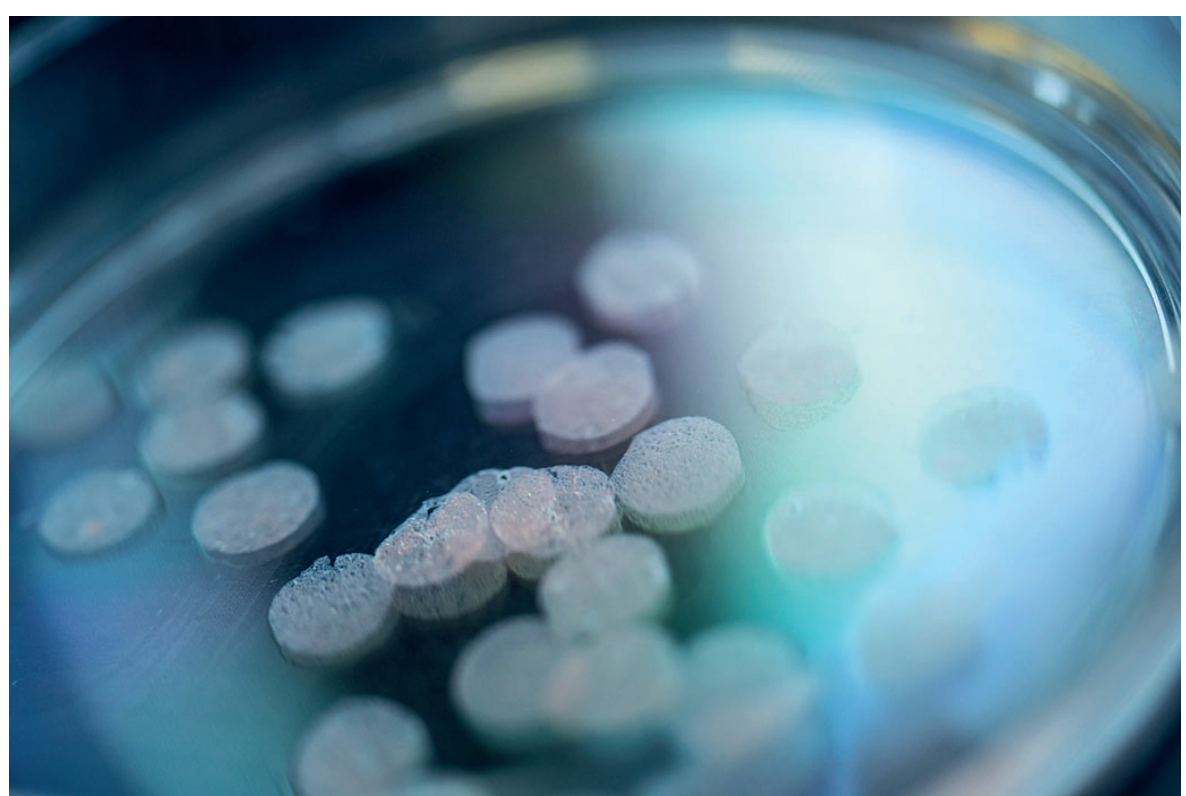

Fraunhofer-Institut für Toxikologie und Experimentelle Medizin

\section{Infektionen und Resistenzen bekämpfen}

Laut Robert-Koch-Institut waren die Influenza-Vakzinen für das Jahr 2018/2019 nur zu etwa 20\% effektiv. Neue antiinfektive Wirkstoffe und Therapiekonzepte soll nun das Projekt iCAIR ${ }^{\circledR}$ (Fraunhofer International Consortium for Anti-Infective Research) entwickeln, insbesondere für das InfluenzaVirus, das Parainfluenza-Virus, die Bakterien Pseudomonas aeruginosa und Neisseria meningitidis sowie für den Pilz Aspergillus fumigatus. Der Anspruch des Konsortiums: Neue Wirkstoffe sollen schnellstmöglich vom Labor in die präklinische Phase überführt werden.

Das Fraunhofer-Institut für Toxikologie und Experimentelle Medizin ITEM verfügt über spezielle Testsysteme, um Wirkstoffkandi- daten auf ihre Wirksamkeit zu prüfen: Die Forscherinnen und Forscher verwenden lebende Lungengewebsschnitte, die aus menschlichen Lungen oder Lungenteilen - etwa nach einer Tumor-Operation - gewonnen wurden. Mit diesen Precision-Cut Lung Slices, kurz PCLS, entwickeln sie Infektionsmodelle mit Bakterien und Viren, um die Vorgänge direkt im menschlichen Lungengewebe untersuchen zu können.

«Wir haben gezeigt, dass wir die PCLS nutzen können, um die Infektion von menschlichem Lungengewebe mit dem Influenza-Virus nachzustellen. Wir haben vielversprechende Wirkstoffkandidaten unserer australischen iCAIR ${ }^{\circledR}$-Partner getestet, die die Virusmenge verringern, weil sich das
Etwa $500 \mu m$ dünn sind die Lungengewebsschnitte, mit denen die Forscher Infektionen im menschlichen Gewebe untersuchen. () Fraunhofer ITEM / Ralf Mohr
Virus nicht mehr so gut ausbreiten kann», sagt Dr. Jana Führing, Wissenschaftlerin am Fraunhofer ITEM. Die Wirkstoffkandidaten werden aktuell weiter optimiert. Langfristiges Ziel des iCAIR ${ }^{\circledR}$-Konsortiums ist es, mit den neuen Methoden der gezielten Wirkstoffentwicklung und -testung einen Paradigmenwechsel in der Forschung für neue Antiinfektiva einzuleiten und seine Forschungs- und Entwicklungsplattform externen Partnern zur Verfügung zu stellen. 
Fraunhofer-Einrichtung für Marine Biotechnologie und Zelltechnik

\section{Schnelltest für die Asthma-Diagnose}

Ein Forscherteam an der Fraunhofer-Einrichtung für Marine Biotechnologie und Zelltechnik EMB entwickelt gemeinsam mit Hightech-Unternehmen einen Schnelltest, der zur Asthma-Diagnose nur einen Tropfen Blut benötigt.

«Bei Asthma ist die Bewegung der Immunzellen stark verlangsamt, wenn sie einen Entzündungsreiz erfahren», erklärt Dr. Daniel Rapoport, Leiter der Arbeitsgruppe Zellprozessierung an der Fraunhofer EMB. Dieses Wissen macht sich die Forschergruppe bei der Entwicklung des Testsets zunutze. Die Idee dahinter ist, die Immunzellen aus dem Tropfen Blut unter einem eigens entwickelten holografischen Mikroskop etwa 90 Minuten lang zu beobachten und anhand ihrer Bewegungsmuster einzuschätzen, ob eine Asthmaerkrankung vorliegt. Das Mikroskop, auch als Zellscanner bezeichnet, ermöglicht ein automatisches,

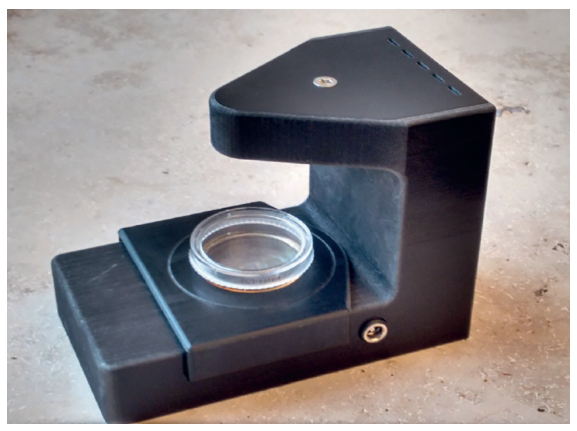

Das holografische Mikroskop ermöglicht ein automatisches, dreidimensionales Tracking der Immunzellen in Echtzeit. @ Fraunhofer EMB

dreidimensionales Tracking der Zellen in Echtzeit. Eine entscheidende Rolle kommt der Künstlichen Intelligenz (KI) zu, die in den komplexen Bewegungsmustern tausender Zellen charakteristische Muster erkennt. «Mit unserer Methode lassen sich auch andere Krankheiten analysieren. Dies gilt ins- besondere für Autoimmun- und chronisch entzündliche Erkrankungen wie Morbus Crohn, Colitis Ulcerosa und Rheuma. Hier sind die Diagnosen langwierig und lassen sich mit einem angepassten Schnelltest deutlich beschleunigen», sagt der Lübecker Forscher. «Erste Tests sind erfolgreich abgeschlossen. Die Bildauswertung ergab, dass unser holografisches Mikroskop einem Hochleistungsmikroskop überlegen ist.» Rapoport und seine Projektpartner optimieren derzeit die Hardware sowie das Verfahren. Fernziel ist, die individuelle Ausprägung von Asthmaerkrankungen zu erkennen, um einen personalisierten Behandlungsplan entwickeln zu können.

\section{Fraunhofer} EMB

www.emb.fraunhofer.de/

Bundesinstitut für Risikobewertung (BfR)

\section{Kohlenmonoxid-Vergiftung: Viele wissen zu wenig}

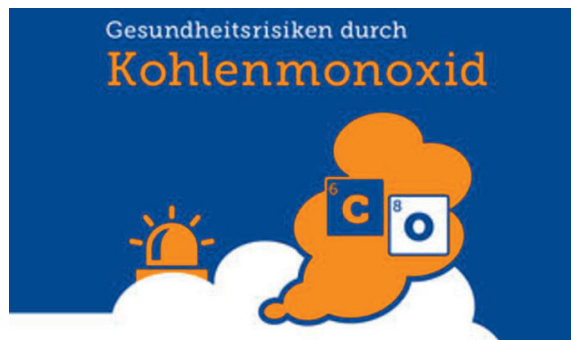

Auch beim Rauchen von Shishas entsteht Kohlenmonoxid. @ Bundesinstitut für Risikobewertung

Das BfR hatte mehr als 1000 Personen in Deutschland telefonisch zu Bekanntheit, Wahrnehmung, Wissen und Präventionsverhalten im Zusammenhang mit Vergiftungsrisiken befragen lassen.
Im Fokus der Befragung stand besonders auch das Wissen zur Vergiftungsgefahr durch das Gas Kohlenmonoxid (CO). Vielen Befragten (82\%) war bewusst, dass Kohlenmonoxid zu Vergiftungen führen kann und die überwiegende Mehrheit gab an, sich der meisten Symptome, wie Kopfschmerzen, Schwindel oder Übelkeit bewusst zu sein. Allerdings glaubt mehr als ein Drittel der Befragten fälschlicherweise, man könne Kohlenmonoxid am Geruch, an dunklem Rauch oder durch den Alarm von Rauchmeldern erkennen.

Auch wissen nur 59\%, dass das Rauchen von Wasserpfeifen (Shishas) in geschlossenen Räumen zu Vergiftungen führen kann.
Und nur 20\% der Befragten ist bewusst, dass Holzpellets beim Lagern Kohlenmonoxid ausgasen.

Die Ergebnisse der Befragung hat das BfR sehr anschaulich in einem Booklet «Gesundheitsrisiken durch Kohlenmonoxid» (PDF) zusammengefasst. Darin finden Leserinnen und Leser auch eine Checkliste zum Schutz vor Kohlenmonoxid-Vergiftungen. Es ist kostenlos abzurufen unter www.bfr. bund.de/cm/350/gesundheitsrisiken-durchkohlenmonoxid.pdf.

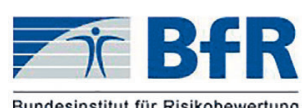

www.bfr.bund.de 


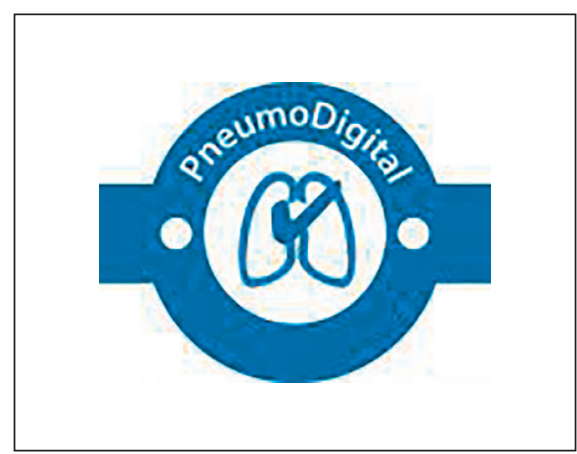

So wird das PneumoDigital-Siegel vergeben: Der App-Hersteller bewirbt sich um das Siegel und füllt eine Selbstauskunft zu der App aus. Das Zentrum für Telematik und Telemedizin in Bochum (ZTG) nimmt eine technische Überprüfung vor und erstellt einen Bericht. Die App-Tester (Ärzte und Patienten) von PneumoDigital führen ihre individuelle Bewertung durch. In einer Telefonkonferenz, an der alle Tester teilnehmen können, wird überprüft, ob die App alle wichtigen Kriterien erfüllt. Die Ergebnisse der Tester werden in einem Fazit zusammengefasst.
Die Deutsche Atemwegsliga e.V., die Deutsche Gesellschaft für Pneumologie und Beatmungsmedizin e.V. und der Verband pneumologischer Kliniken e.V. haben ein Positionspapier zur Digitalisierung im Gesundheitswesen veröffentlicht.

In der Verwendung digitaler Hilfsmittel sehen sie eine Ergänzung in der klassischen Beziehung und Kommunikation zwischen Ärztin/Arzt und Patientin/Patient. Es bietet sich die Chance, die Versorgung zu verbessern und durch potenzielle Entlastung von Routine mehr Zeit für schwerer und/oder akut Erkrankte zu gewinnen. Auch für die transsektorale Patientenversorgung, insbesondere bei chronisch und schwer Erkrankten, sowie Patienten mit seltenen Erkrankungen lässt sich ein Potenzial erkennen. Zusätzliche Vorteile für die Versorgung wer- den im ländlichen Raum gesehen. Dazu müssen solche Anwendungen ausreichend evaluiert und bei Effektivität auch honoriert werden. Datensicherheit, Datenhoheit und Datenschutz müssen gewährleistet und Haftungsfragen geklärt sein.

Ein weiteres und wichtiges Thema ist die Orientierung. Zertifizierte Angebote wie z.B. das PneumoDigital-Siegel der Deutschen Atemwegsliga können Patientinnen und Patienten bei der Auswahl geeigneter Angebote unterstützen.

Das vollständige Positionspapier ist online abrufbar.

dia

Deutsche Atemwegsliga e.V.

In der Deulschen Gesellschaft für Pneumologie

www.atemwegsliga.de

\section{Deutsche Allianz Nichtübertragbare Krankheiten (DANK) \\ Wissenschaftler fordern schnelles Tabakwerbeverbot ohne Ausnahmen}

Das Bündnis «Deutsche Allianz Nichtübertragbare Krankheiten» (DANK) aus 22 Wissenschaftsorganisationen drängt auf eine schnelle Erarbeitung und Verabschiedung eines Gesetzes zum Tabakwerbeverbot. Seit Wochen verzögert eine Gruppe von Abgeordneten innerhalb der CDU/CSUFraktion den Entscheidungsprozess.

«Wir begrüßen sehr, dass sich nun auch Ernährungsministerin Julia Klöckner für ein Werbeverbot einsetzt, das E-Zigaretten einschließt», sagt Dr. Ute Mons vom Deutschen Krebsforschungszentrum in Heidelberg. «Ein Außenwerbeverbot für E-Zigaretten ist gesundheitspolitisch sinnvoll», jedoch dürfe die Debatte um die Einbeziehung der E-Zigaretten in das Werbeverbot nicht dazu benutzt werden, den Gesetzentwurf weiterhin zu verzögern, so Mons. Studien zur Wirkung von E-Zigaretten-Werbung aufjunge Menschen weisen darauf hin, dass diese die Bereitschaft von Jugendlichen steigert, solche Produkte auszuprobieren [1]. Diesen Zusammenhang findet auch eine Studie mit 6500 deutschen Teenagern [2]. Zudem zeigt eine aktuelle Studie aus den USA, welche gesundheitlichen Langzeitfolgen der Konsum von E-Zigaretten hat [3]. Mehr als die Hälfte der Bevölkerung befürwortet ein vollständiges Werbeverbot für E-Zigaretten - sogar fast jeder zweite Raucher [4]. Laut Tabakrahmenkonvention der Vereinten Nationen und Beschluss des Bundestages hätte die Regierung schon bis
2010 ein umfassendes Tabakwerbeverbot erlassen müssen. Eine weitere Verzögerung würde die Tabakindustrie wie in den vergangenen Jahren dafür nutzen, noch mehr Werbung für ihre Produkte zu machen.

\section{Literatur}

1 Collins L et al.: Nicotine Tob Res 2019;21:14-24.

2 Hansen J, Hanewinkel R, Morgenstern M: ERJ Open Research 2019;4.

3 Bhattan et al.: Am J Prev Med 2019;000(000):1-9. E-Pub ahead of Print.

4 Kastaun S, Kotz D: Bundesgesundheitsblatt, Gesundheitsforschung,Gesundheitsschutz.2019;62(11):13911396.

\section{DANK Deutsche Allianz Nichtübertragbare Krankheiten}


Schweizerisches Tropen- und Public Health-Institut

Verbesserung des Tuberkulose-Screenings in abgelegenen Gebieten

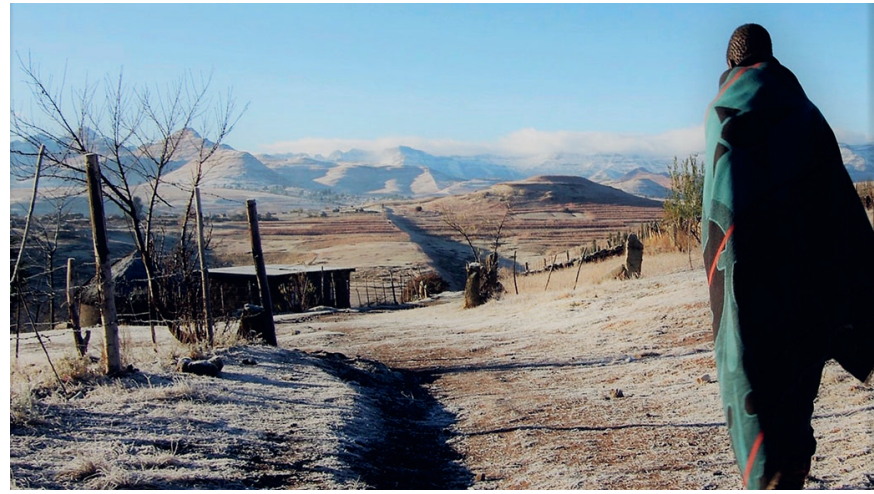

Tuberkulosepatienten finden und behandeln ist in abgelegenen Gebieten eine große Herausforderung. (Foto: Niklaus Labhardt / Swiss $\mathrm{TPH})$

Das Schweizerische Tropen- und Public Health-Institut (Swiss TPH) kündigt zusammen mit internationalen Partnern den Start des 4-jährigen TB TRIAGE+-Projekts an. TB TRIAGE+ wird neue diagnostische Ansätze evaluieren, um die aktive Fallsuche von TB-
Patienten in abgelegenen Gebieten im südlichen Afrika effizienter und kostengünstiger zu gestalten.

Das TB TRIAGE+-Projekt wird die Exaktheit, Wirkung und Kosteneffizienz neuer Fallsuch-Methoden auf Gemeindeebene eru- ieren, um potenzielle Tuberkulosepatienten besser zu triagieren. Das Herzstück von TB TRIAGE+ ist eine groß angelegte, clusterrandomisierte klinische Studie in den ländlichen Gemeinden Lesotho und KwaZuluNatal in Südafrika, die stark von TB- und HIVEpidemien betroffen sind.

Neben der großen klinischen Studie will TB TRIAGE+ auch eine Reihe von Forschungsfragen im Zusammenhang mit Triage-Tests und der aktiven TB-Fallsuche beantworten.

\section{Klinikum der Universität München \\ Neuer Wirkstoff BTZ-043 gegen Tuberkulose erstmals Patienten verabreicht}

Das Tropeninstitut am Klinikum der Ludwig-Maximilians-Universität (LMU) München führt derzeit unter der Leitung von Professor Michael Hoelscher eine klinische Studie durch. Seit Mitte November werden darin erstmals Tuberkulose-Patientinnen und -Patienten mit der neuen Substanz BTZ-043 in Kapstadt, Südafrika, behandelt. Eine enorme Herausforderung bei der Behandlung von Tuberkulose ist das vermehrte Auftreten resistenter Keime, gegen die nur noch wenige Antibiotika helfen. Die
WHO setzt daher große Hoffnungen auf die Entwicklung von Medikamenten, die auf neuen Wirkstoffen basieren. Die Prüfsubstanz BTZ-043, ein Benzothiazinon, ist solch ein neuer Wirkstoff.

Das internationale Forschungsteam befindet sich mit dem Start der Studie in Kapstadt an einem entscheidenden Punkt: Eine in Deutschland durchgeführte Phase-I-Studie hatte eine gute Verträglichkeit von BTZ043 gezeigt. Erweist sich der Wirkstoff in der jetzt laufenden Studie (Phase II) als sicher und wirksam, wäre dies ein großer Schritt in der Entwicklung eines neuen Medikaments. Dieses könnte in absehbarer Zukunft eines der herkömmlichen, häufig resistenzbehafteten Antibiotika in einer Kombinationstherapie ersetzen und die Behandlungsdauer der Tuberkulose deutlich verkürzen.

KLINIKUM OER UNIVERSITAT MUNCHEN

www.klinikum.uni-muenchen.de 